\title{
Epigenetics and the Control of the Collecting Duct Epithelial Sodium Channel
}

\author{
Bruce C. Kone, M.D. [Professor of Medicine] \\ Division of Renal Diseases and Hypertension, Department of Internal Medicine, The University of \\ Texas Medical School at Houston, 77030
}

\begin{abstract}
The apical membrane epithelial $\mathrm{Na}^{+}$channel subunit $(\mathrm{ENaC})$ in series with the basolateral $\mathrm{Na}^{+}$/ $\mathrm{K}^{+}$-ATPase mediates collecting duct $\mathrm{Na}^{+}$reabsorption. Aldosterone induces aENaC gene transcription, which appears to be rate limiting for $\mathrm{ENaC}$ activity in this segment. While this response has long been assumed to be solely the result of liganded nuclear hormone receptors trans-activating $\mathrm{a} E \mathrm{NaC}$, epigenetic controls of basal and aldosterone-induced transcription of $\alpha E N a C$ in the collecting duct have recently been described. These epigenetic pathways involve dynamic nuclear repressor complexes targeted to specific subregions of the $\alpha E N a C$ promoter and consisting of the histone methyltransferase disrupter of telomeric silencing (Dot)1a together with the transcriptional factor Af9 or the NAD-dependent protein deacetylase Sirt1, key co-regulatory proteins, including serum- and glucocorticoid-induced kinase (Sgk1) and the putative transcription factor Af17, and targeted chromatin modifications. The complexes, through the action of Dot1a, maintain chromatin associated with the $\mathrm{a} E N \mathrm{Na}$ promoter in a stable hypermethylated state, constraining aENaCtranscription under basal conditions. Aldosterone and Sgk1, itself, activate $\mathrm{a} E N a C$ transcription in large part by disrupting or diminishing the Dot1a-Af9 and Dot1a-Sirt1 complexes and their effects on chromatin. Mouse models indicate potential roles of the Dot1a pathways in renal salt excretion and hypertension.
\end{abstract}

\section{Keywords}

gene transcription; chromatin; epithelial sodium channel; histone; blood pressure

\section{INTRODUCTION}

The epithelial sodium channel $(\mathrm{ENaC})$ is expressed in the apical membrane of salt-absorbing epithelia of the aldosterone-sensitive distal nephron, where it constitutes the rate-limiting step in active $\mathrm{Na}^{+}$absorption1. Hence, $\mathrm{ENaC}$ plays a major role in the regulation of extracellular volume homeostasis and blood pressure ${ }^{2,3}$. In the kidney, $\mathrm{ENaC}$ is expressed in the principal cells of the collecting duct, where it participates in the final regulation of urinary sodium ( $\mathrm{Na}$ ) excretion. The physiological importance of $\mathrm{ENaC}$ is illustrated by the

() 2013 Elsevier Inc. All rights reserved.

Full Address for Corresponding Author: Bruce C. Kone, M.D., FACP, FCP, FAHA, FASN, FAAAS, Division of Renal Diseases and Hypertension, The University of Texas Medical School at Houston, 6431 Fannin, MSB 5.124, Houston, TX 77030, Telephone: 713 500-6880, Bruce.C.Kone@uth.tmc.edu.

Publisher's Disclaimer: This is a PDF file of an unedited manuscript that has been accepted for publication. As a service to our customers we are providing this early version of the manuscript. The manuscript will undergo copyediting, typesetting, and review of the resulting proof before it is published in its final citable form. Please note that during the production process errors may be discovered which could affect the content, and all legal disclaimers that apply to the journal pertain.

Conflict of interest: none 
findings in humans of $E N a C$ gain-of-function and loss-of-function mutations resulting in monogenic hypertensive (Liddle's syndrome ${ }^{4}$ ) and hypotensive (pseudohypoaldosteronism type $1^{5}$ ) diseases, respectively. ENaC is composed of three homologous subunits- $\alpha, \beta$, and $Y$-encoded by the Scnn1a, $S c n n 1 b$ and $S c n n 1 c$ genes (I will refer to $S c n n 1 a$ as aENaC). The $\alpha$ subunit mediates ion translocation, whereas the $\beta$ and $\gamma$ subunits serve regulatory roles. Complex regulatory controls including biophysical, transcriptional, and posttranslational effects choreograph the ultimate function of $\mathrm{ENaC}$ at the apical membrane of collecting duct principal cells 6

Under basal conditions, aENaC gene transcription is constrained but active and poised for maximal induction by aldosterone and other stimuli, including the immediate early gene Sgk $1^{7}$. Increased circulating aldosterone, as seen in primary hyperaldosteronism, results in expansion of extracellular fluid volume and hypertension, in large part via activation of $\mathrm{ENaC}^{8}$. Experimentally, aldosterone administration or hyperaldosteronism induced by a low- $\mathrm{Na}^{+}$diet increases $\alpha E N a C$ gene transcription, without increasing $\beta$-or $\gamma$-subunit expression ${ }^{9}$, in the cortical collecting duct. Since this transcriptional response appears to be rate limiting for $\mathrm{ENaC}$ activity in this segment, and since aldosterone does not alter $\mathrm{aENaC}$ mRNA turnover ${ }^{8}$, much investigative attention has been placed on mechanisms for transcriptional activation of $a E N a C$. The classical mechanism of aldosterone action involves binding to the cytoplasmic mineralocorticoid receptor (MR), which functions as a liganddependent transcription factor for target genes containing cognate hormone response elements (HRE, Figure 1). Indeed, promoter-reporter studies of the murine $a E N a C$ gene in collecting duct cells established the functional importance of such an HRE residing at -811 in the aldosterone response ${ }^{10}$. However, mice with connecting tubule/collecting duct (CNT/ $\mathrm{CD})$-specific ablation of the MR did not develop the severe salt-wasting phenotype ${ }^{11}$ observed with targeted ablation of $\mathrm{aENaC}$ in these same segments ${ }^{12}$, suggesting that MRindependent pathways in aENaC regulation compensated for the response. Recent work indicates that one such alternative pathway for aldosterone control of $\mathrm{aENaC}$ transcription is an MR-independent epigenetic pathway whose central effector is the histone methyltransferase (HMT) disruptor of telomere silencing (Dot)1. This review summarizes progress and open questions with regard to this pathway.

Dot1: structure, function, and role in epigenetic silencing of ENaC. Eukaryotic genomic DNA is compacted with histone and non-histone proteins into a dynamic polymer termed chromatin. The basic repeating unit of chromatin is the nucleosome, which includes two copies of each of the four core histones H2A, H2B, H3, and H4 wrapped by 146 bp of DNA. Each core histone contains a structured domain, the histone fold, and two tails. Reversible changes in chromatin organization, through site-specific post-translational modifications of histones, position- and modification-specific protein interactions, biochemical crosstalk between modifications, and histone turnover dynamically regulate gene expression with in any given cell type ${ }^{13}$. These epigenetic modifications allow heritable and potentially reversible changes in gene functioning to occur without altering the DNA sequence, thus extending the information content of the genetic code. Genes are actively transcribed when the chromatin is open, but silent when the chromatin is condensed. Combinatorial interactions of various histone modifications likely dictate the "ON" or "OFF" mode of transcription for specific genes.

Methylation of arginine (R) or lysine (K) residues of histone represents one such covalent histone modification ${ }^{14}$. Arginine methylation exists in mono-and di-methylation states, whereas lysine methylation can occur as mono-(abbreviated as me1), di- (me2), and trimethylation (me3) states. These different methylation states of histones often lead to different functions. Three subfamilies of HMTs mediate histone methylation. The CARM1/ PRMT1 (co-activator-associated arginine methyltransferase, protein arginine 
methyltransferase) family of HMTs is recruited as co-activators to gene promoters and target histones H3 or H4 ${ }^{15}$. The SET (Suppressor of Variegation3-9, Enhancer of Zeste, and Trithorax) domain family of lysine HMTs silences genes via histone H3 lysine methylation ${ }^{16}$. The most recently described, third class of evolutionarily conserved HMTs, represented by yeast Dot 1 and its homologs in other organisms ${ }^{17-19}$, lacks a SET domain but contains a catalytic methylase fold similar to that of class I S-adenosyl-L-methioninedependent methylases ${ }^{18,20}$. Whereas most covalent modifications occur histone tails, Dot1 specifically methylates K79 in the histone H3 globular domain (Figure $2^{19,}{ }^{20}$. Dot1 can catalyze mono-, di-, and trimethylation in a nonprocessive (that is, non-stepwise) manner. Since knockout of Dot1 in mice results in complete loss of H3 K79 methylation ${ }^{21}$, Dot1 appears to be solely responsible for this posttranslational modification. Thus identification of the H3 K79 methylation mark can be assumed to arise through the action of Dot1. Although Dot1 was originally identified in a genetic screen in yeast seeking overexpressed genes that disrupt telomeric silencing ${ }^{22}$, subsequent studies have implicated H3 K79 methylation in regulating transcription at both heterochromatin and euchromatin, in cell cycle regulation, and in the DNA damage response ${ }^{21}, 23,24$. Mammalian DOT1L plays key roles in embryogenesis, hematopoiesis, heart function, kidney function, and in MLL-related leukemogenesis ${ }^{23}$. In addition to its roles in the control of $\mathrm{ENaC}$ in the collecting duct, intriguing new data has implicated Dot1 in the control of collecting duct water channel ${ }^{25}$ and endothelin expression ${ }^{26}$, and mesangial cell connective tissue growth factor expression $^{27}$.

The orthologous human gene, termed DOT1L, consists of 28 exons on chromosome 19 that encode a 1547 amino acid protein. Structurally, the N-terminus contains a lysine-rich DNAbinding domain, whereas the C-terminal domain exhibits similarities to the SAM motif and lysine-rich region $n$ the catalytic domain ${ }^{20}$. The highly homologous murine Dot 1 gene is comprised of 28 exons on chromosome 10qC1, with exons 24 and 28 subdivided into two and four sections, respectively ${ }^{28}$. In mice, five Dot 1 transcript variants (Dot1a-Dot1e) arise by alternative splicing in exons 3, 4, 12, 24, 27 and 28. The 1540 amino acid protein Dot1a most closely resembles human DOT1L, sharing $84 \%$ amino acid identity. Dot1b contains 1114 amino acids contains an internal deletion and is truncated at its $\mathrm{N}$ - and $\mathrm{C}$-termini, whereas Dot1c-Dot1e are incomplete at the 5'-end. The functional relevance of the Dot 1b-e splice variants is unknown. Northern-blot analysis with probes directed at the conserved methyltransferase domain or the Dot1a-coding region detected 7.6 and $9.5 \mathrm{~kb}$ transcripts in multiple mouse tissues, including the kidney, but only the $7.6 \mathrm{~kb}$ mRNA was evident in mIMCD3-collecting duct cells ${ }^{28}$. Colocalization studies confirmed that Dotla was expressed in collecting duct principal cells as well as other renal cell types ${ }^{28}$. Transfection of Dot1aenhanced green fluorescent protein (EGFP) constructs into human embryonic kidney (HEK)-293T or mouse inner medullary collecting duct (mIMCD) 3 cells increased the methylation of histone $\mathrm{H} 3 \mathrm{~K} 79$ but not of $\mathrm{H} 3 \mathrm{~K} 4$, $\mathrm{K} 9$ or $\mathrm{K} 36^{28}$, consistent with the known target histone residue specificity of Dot1.

An important early clue to the functional role of Dot1 in regulating $E N a C$ subunit gene transcription was the finding that aldosterone treatment of mIMCD3 cells lowered Dot1a mRNA levels and resulted in hypomethylated H3 K79 in bulk histones ${ }^{28}$, thereby identifying Dot1a as the first known aldosterone-sensitive chromatin modifier. This result led to the hypothesis that in the absence of aldosterone, Dot1a-mediated histone H3 K79 hypermethylation at aldosterone-sensitive target promoters, such as that of $\mathrm{a} E N \mathrm{NC}$, might alter local chromatin structure so as to repress transcription, and that aldosterone-dependent suppression of Dot1a expression and activity would allow relative hypomethylation of histone H3 K79, a more favorable chromatin configuration, and de-repression of transcription (Figure 3). By manipulating Dotla expression levels by overexpression or RNA silencing in mIMCD3 cells and measuring the consequences on endogenous $\mathrm{aENaC}$ 
mRNA levels and the activity of aENaC promoter-reporter constructs, this hypothesis was proved. Overexpression of EGFP- tagged Dot1a, but not a methyltransferase-dead mutant, hypermethylated histone $\mathrm{H} 3 \mathrm{~K} 79$ at the endogenous a $E N a C$ promoter, inhibited endogenous aENaC $m R N A$ expression, and abrogated the activity of a stably integrated aENaC promoter-luciferase construct in mIMCD3 cells ${ }^{29}$. The inhibitory effect of Dot1a on basal a $E N a C$ transcription was substantial, since Dotla silencing resulted in three-fold higher endogenous a $E N a C$ mRNA levels and tripled activity of the stably integrated $a E N a C$ promoter-reporter construct ${ }^{29}$. Also consistent with this hypothesis, aldosterone treatment resulted in histone $\mathrm{H} 3 \mathrm{~K} 79$ hypomethylation at specific sites in the $\mathrm{a} E N a C 5$ '-flanking region, enhanced activity of the stably integrated $a E N a C$ promoter-luciferase construct, and increased expression of endogenous aENaC $\mathrm{mRNA}^{29}$ in mIMCD3 cells. Similar results regarding Dot 1a suppression of aENaC gene expression were reported in HEK 293T cells $\mathrm{s}^{30}$ and $\mathrm{M} 1$ collecting duct cells ${ }^{31}$. In these latter cell types, the ability of Dot1a overexpression to inhibit benzamil-sensitive $\mathrm{Na}^{+}$current density, the functional readout of ENaC-mediated $\mathrm{Na}^{+}$transport, was also established ${ }^{30,31}$. Most recently, renal $a E N a C$ gene expression was evaluated in mice harboring CNT/CD-specific knockout of Dot $1^{32}$. As predicted from the cell culture data, and despite the fact that they unexpectedly possessed approximately $20 \%$ fewer principal cells, the mutant mice exhibited 36\% greater levels of a $E N a C$ mRNA compared to their controls on a normal salt diet ${ }^{32}$. Presumably this increment in aENaC gene expression is the result of a lack of Dot1a-mediated repression of aENaC transcription. Analysis of $\alpha E N a C$ expression in this mouse model under variations in salt intake or when subjected to aldosterone treatment will be important confirmations of the derepression hypothesis. Finally, the question of whether Dot1a coordinately regulates the other $\mathrm{ENaC}$ subunits remains incompletely defined. Dot1 overexpression inhibited mRNA expression not only of $\alpha E N a C$, but also of $\beta E N a C$ and $\gamma \mathrm{ENaC}$ in HEK 293T cells and mIMCD $3^{31}$ cells. However, in the mice with CNT/CD-specific ablation of Dot1, only a $E N a C$ expression was affected ${ }^{32}$.

The transcription factor Af9: targeting Dot1a to the $a E N a C$ promoter. Since it had been established that Dot1a does not exhibit sequence-specific DNA-binding activity that would allow it to directly bind the $a E N a C$ promoter, a key mechanistic question was whether Dot1a partnered with one or more DNA-binding proteins to allow sequence-specific DNAbinding activity for the complex at specific regions of the $a E N a C$ promoter. The transcription factor Af9, which was subsequently shown to serve this purpose, was initially identified in a yeast two-hybrid screen of a mouse kidney cDNA library ligated to the GAL4 activation domain using Dotla as bait ${ }^{33}$. The interaction was verified in coimmunoprecipitation assays, mammalian two-hybrid assays, and GST pull-down assays ${ }^{34}$. Structure-binding analysis of the protein partners established that amino acids 479-659 of Dot 1a and amino acids 397-557 of Af9 were necessary and sufficient for the interaction ${ }^{35}$. Immunolocalization studies demonstrated nuclear expression of Af9 in various renal cell types, including principal cells of the cortical collecting duct ${ }^{35}$.

Af9 was initially identified as one of more than fifty fusion partners of the mixed lineage leukemia (MLL) gene that result from translocations at the $11 \mathrm{q} 23$ locus $^{36}$ in patients with aggressive leukemias. Af9 was also shown to play important roles in anterior homeotic transformations during mouse development ${ }^{8,37}$ and in neurodevelopmental diseases ${ }^{38}$, as well as in canonical Wnt signaling in mIMCD3 cells ${ }^{39}$. The N-terminus of Af9 contains a YEATS domain, which is found in a variety of chromatin-modifying and transcription complexes. Indeed, sequential chromatin immunoprecipitation (ChIP) assays confirmed that Af9 and Dot1a co-associate with chromatin at specific subregions of the $\mathrm{aENaC \text {promoter }}$ (Figure 1): approximately $75 \%$ of the basal Dot 1a-Af9 association occurred at the $-57 /+439$ "R3" subregion of aENaC, whereas roughly $15 \%$ occurred more distally at the R2 subregion, and only small, but measurable amounts occurred at the R0 and R1 subregions ${ }^{40}$. 
Transfection studies in mIMCD3 cells established the functional consequences of Dot1aAf9 interaction with respect to aENaC transcription. Af9 overexpression resulted in enhanced Dot1a-mediated hypermethylation of H3 K79 at specific subregions of the endogenous $\alpha E N a C$ promoter and suppressed endogenous $\alpha E N a C$ mRNA expression and a $E N a C$ promoter-luciferase constructs in a manner synergistic with that of Dot $1 \mathrm{a}^{33}$. Similar results regarding Dot1a/Af9 suppression of a $E N a C$ gene expression were reported in HEK 293T cells ${ }^{30}$ and M1 collecting duct cells ${ }^{35}$. Structure-function, ChIP, and gel shift assays identified $+78 /+92$ of the aENaC promoter as a functional cis-element for Af9 and for the Af9/Dot 1a complex ${ }^{32}$ (Figure 1). mIMCD3 cell lines expressing a $E N a C$ promoter-reporter constructs harboring mutations of this element demonstrated greatly reduced association of Af9 and Dot1 a by ChIP/qPCR, higher basal aENaC promoter activity, and impaired Dot1amediated inhibition in trans-repression assays ${ }^{32}$. Collectively, these studies supported a model in which Dot1a/Af9 complexes are specifically targeted to the +78/+92 Af9 element (and likely, to a far lesser extent, other regions) of the $\alpha E N a C$ promoter to mediate local histone $\mathrm{H} 3 \mathrm{~K} 79$ hypermethylation and thereby constrain basal aENaC transcription (Figure 3). Af9 knockout mice do not survive, so mouse models to test Af9 deficiency on aENaC expression have been lacking.

Nuclear-cytoplasmic trafficking in control of Dot1 action on ENaC transcription. To effect epigenetic control of $\alpha E N a C$ transcription, Dot1a and Af9 must reside in the nucleus. Recent studies have elucidated mechanisms underlying control of Dot1a and Af9 nuclear localization. Dot1a contains three nuclear localization signals (at amino acids 393-416, 1089-1112, and 1165-1172) that are required for nuclear distribution of Dot1a and for its ability to mediate $\mathrm{a} E N a C$ transcriptional repression ${ }^{35}$. In transient transfection assay, green fluorescent protein-Dot1a fusion proteins harboring deletion of these NLSs localized almost exclusively in the cytoplasm of HEK 293T cells ${ }^{35}$. Moreover, these mutants failed to suppress aENaC expression in M1 collecting duct cells ${ }^{35}$. The nuclear distribution of Af9 appears to require its direct and specific interaction with the heat shock protein Hsp90 as part of an Hsp90-Hsp70-p60/Hop chaperone complex ${ }^{41}$ (Figure 4). Pharmacologic inhibition or siRNA-mediated depletion of Hsp90 results in Af9 redistribution from the nucleus to the cytoplasm, accompanied by decreased Af9 occupancy at the aENaC promoter $^{41}$.

Additional yeast two-hybrid screening identified ALL-1 partner at 17q21 (Af17) (which, like Af9, is an MLL fusion partner) as another Dot1a-interacting protein $^{30}$ (Figure 2). Tissue distribution studies established that it, like Dot1 and Af9, was expressed in the nuclei of collecting duct principal cells, among other renal cell types ${ }^{42}$. Although Af17 was known to play a role in cell cycle progression and to serve as a downstream target of the $\beta$-catenin/ $\mathrm{T}$-cell factor pathway ${ }^{43}$, little was known about its specific roles as a transcriptional regulator until transfection experiments in HEK 293T cells ${ }^{30}$ and M1 collecting duct cells ${ }^{31}$ established that Af17 (via its amino acids 635- 786) competes with Af9 for binding to the Af9 binding domain of Dot1a (Figure 4). Overexpression of AF17, presumably by displacing Af9 from Dot1, promoted leptomycin B (a nuclear export inhibitor)-sensitive redistribution of Dot 1a from the nucleus to the cytoplasm, resulting in hypomethylation of $\mathrm{H} 3 \mathrm{~K} 79$ in bulk histones, and increased aENaC mRNA levels, the activity of an aENaC promoter-reporter construct, and benzamil-sensitive $\mathrm{Na}^{+}$current density ${ }^{31}$. Interestingly, the effects of Af9 to suppress, and Af17 to enhance, gene expression extended to the $\beta$ - and $\gamma$ $\mathrm{ENaC}$ subunits in these cell types ${ }^{31}$. Treatment with leptomycin B or siRNA-mediated depletion of Af17 resulted in nuclear accumulation of Dot $1 \mathrm{a}^{31}$. Most impressively, global inactivation of Af17 in mice results in lower blood pressure, increased sodium excretion (despite mildly increased plasma concentrations of aldosterone), and dramatic (>80\%) reductions in renal $\alpha E N a C$ mRNA levels, and to a lesser ( 25\%) extent $\beta E N a C$ and $\gamma \mathrm{ENaC}$ mRNA levels compared to wild type controls on a normal sodium diet ${ }^{44}$. The mutant mice 
also exhibited greater dimethylation of histone H3 K79 and lower numbers of functional $\mathrm{ENaC}$ channels in the distal nephron on a normal salt diet ${ }^{44}$. Unfortunately, since there is no available antibody that specifically detects endogenous Af17 in immunoblotting, immunohistochemistry, immunofluorescence, or ChIP assays, it remains an open question as to whether Af17 regulates nuclear-cytoplasmic shuttling of the Dot1a-Af9 complex bound to the $\mathrm{a} E N a C$ promoter or free from it.

Dot1a/Sirt1 complexes and basal control of aENaC transcription. In addition to Af9/Af17, Dot1a also interacts with Sirt1, an NAD+-dependent deacetylase, to regulating a $E N a C$ gene expression. Sirt1 is known to target histone and select non-histone proteins, and to play a role in gene silencing, metabolism, aging, and oxidative stress responses ${ }^{45,46}$. Sirt1 was identified in nuclei of collecting duct principal cells of mice ${ }^{47}$. In mIMCD3 cells, Sirt1 overexpression inhibited basal a $E N a C \mathrm{~m} R N A$ expression and a $E N a C$ promoter activity, surprisingly in a deacetylase-independent manner. Co-localization, co-immunoprecipitation, and sequential ChIP studies supported the conclusion that Sirt1 and Dot1a physically interact and co-associate with chromatin associated with specific regions of the aENaC promoter ${ }^{47}$. The fact that coexpression of Sirt1 and Dot1a yielded a greater inhibitory effect on $\mathrm{a} E N \mathrm{NaC}$ promoter activity than expression of either protein alone ${ }^{47}$ suggests that Sirt1 and Dot1a have partially additive, independent inhibitory effects (with that of Sirt1 being presently unknown) on aENaC transcription, or that Sirt1 association with Dot1a amplifies or sustains the latters methyltransferase activity in chromatin, and hence its repression of $\alpha E N a C$ transcription. The latter model that Sirt1 facilitates the basal\% distributive activity of Dot1 for the various H3 K79 methylation states is supported by the fact that Sirt1 knockdown was associated with global H3 K79 hypomethylation and near loss of H3 $\mathrm{K} 79 \mathrm{me} 3$ at the $\mathrm{aENaC}$ promoter compared with controls ${ }^{47}$. The effect of Sirt1 to enhance Dot1a methyltransferase activity predominated at the R1 and R3 subregions ${ }^{47}$ (Figure 1). It remains to be established whether the Dot1a/Sirt1 suppression of basal aENaC expression occurs in the collecting duct in vivo. Moreover, since the Sirt1 effect on Dot1a appears not to be mediated changes in acetylation or ADP ribosylation of histone and/or non-histone targets, the exact mechanism underlying the effect remains to be elucidated.

Aldosterone-mediated de-repression of ENaC. Aldosterone has complex, and temporally distinct actions on $\mathrm{ENaC}$ expression, with both immediate $(<3 \mathrm{~h})$ effects, attributed to increased trafficking or activity of $\mathrm{ENaC}$ in the apical membrane, and delayed actions $(>3 \mathrm{~h}$ ) that involve the synthesis of new $\mathrm{ENaC}$ subunits $1,48,49$. Aldosterone was shown to transiently suppress Dot1a and Af9, but not Af17, mRNA levels in mIMCD3 cells and HEK $293 \mathrm{~T}$ cells ${ }^{28,30,34}$. These results suggested that, by suppressing the abundance of Dot1a and Af9 available to complex at the aENaC promoter, aldosterone might overcome this basal epigenetic repression of $\alpha E N a C$ to stimulate $a E N a C$ transcription (Figure 3). Time-course ChIP/qPCR studies in mIMCD3 cells established the kinetics of aldosterone-dependent Dot1a and Af9 dismissal, epigenetic reprogramming at H3 K79, MR occupancy and RNA pol II enrichment at the R3 subregion of the aENaC promoter $^{32}$. Significant decreases in Af9 and H3 K79me3 occupancy, and enhanced RNA pol II occupancy at R3 were observed beginning at 2 hours of aldosterone treatment ${ }^{32}$. MR occupancy was not observed at any of the time points ${ }^{32}$, consistent with the fact that the known HRE at -811 resides outside R3.

A central question thus remaining was how aldosterone signals to the Dot1a-Af9 complex. Analysis of the Af9 amino acid sequence revealed that Ser459 fell within a consensus sequence for Sgk1 phosphorylation ${ }^{40}$. Aldosterone is known to increase several regulatory proteins, notably serum- and glucocorticoid-induced kinase-1 (Sgk1), which exerts both post-translational and transcriptional influences on $\mathrm{ENaC}^{50}$. It had been presumed that $\mathrm{Sgk} 1$ phosphorylated a transcription factor(s) that stimulated $\alpha E N a C$ transcription. Given the putative Sgk1 phosphorylation site within in Af9, it was hypothesized that aldosterone 
triggered Sgk1 phosphorylation of Af9, which inhibited the Dot1a-Af9 complex in some manner, and thereby released the repressive effects of the complex on aENaCtranscription. Indeed, Sgk1 and Af9 were found to associate with the aENaC promoter. Moreover, Sgk1dependent phosphorylation of Ser435 within Af9 reduced, but did not abolish, the Dot1aAf9 interaction in binding assays, inhibited Dot1a association and consequent H3 K79 hypomethylation at the $\mathrm{a} E N \mathrm{NC}$ promoter, and thereby released the repressive effects of the complex on a-ENaC transcription in miMCD3 cells ${ }^{40}$ (Figure 3). This Sgk1 effect also occurred principally at the R3 subregion: in sequential ChIP/qPCR assays, mIMCD3 cells transfected with an Sgk1 expression construct exhibited an approximately $80 \%$ reduction in Dot1a-Af9 occupancy and H3 K79 methylation at the R3 subregion of the aENaC gene ${ }^{40}$. No changes in H3 K79 methylation were observed at the R2 subregion (the next highest site of Dot1a-Af9 binding under basal conditions) in these assays ${ }^{40}$. The in vivo correlate of these observations was demonstrated in wild type and $S g k 1$ null mice subjected to manipulation of dietary salt intake. As expected, wild type mice maintained on a low-salt diet exhibited coordinately enhanced levels of renal aENaC protein expression and Af9 phosphorylation. Conversely, $S g k 1$ null mice exhibited reduced levels of Af9 phosphorylation and aENaC mRNA levels when placed on a low-salt diet compared with wild-type mice fed the same diet ${ }^{40}$. Based on the data from $S g k 1$ null mice, ENaC expression and mineralocorticoid action in the kidney are partially, but not completely, dependent on Sgk1; thus other mechanisms must be operative and might work in parallel.

Aldosterone also inhibited Sirt1 mRNA expression as it induced aENaC transcription, and Sirt1 overexpression inhibited aldosterone induction of $a E N a C$ transcription in an MRindependent manner ${ }^{47}$. Moreover, since Af9 and Sirt1 and Af9 did not associate in coimmunoprecipitation or sequential ChIP assays ${ }^{47}$, and since and Dot1a-Af9 and Dot1aSirt1 exhibited aldosterone responses at different subregions of the aENaC promoter in ChIP assay ${ }^{40,47}$, the two protein pairs appear to be separate and to co-regulate basal and $\mathrm{a} E \mathrm{NaC}$ transcription. In contrast, aldosterone did not alter Af17 expression levels ${ }^{31}$, and no additive effect on aENaC mRNA expression was observed when Af17 overexpression or depletion was combined with aldosterone treatment ${ }^{31}$, indicating that Af17 abundance apparently does not participate in the aldosterone induction of aENaC transcription in collecting duct cells in culture. However, Af17 overexpression stimulates Sgk1 expression in mIMCD3 cells and M1 cells ${ }^{31}$. Thus, in addition to competing with Af9 to bind Dot1a, Af17 might also facilitate $\alpha E N a C$ de-repression by increasing Sgk1 expression to enhance Sgk1-mediated Af9 phosphorylation. However, experimentally increasing plasma aldosterone levels further in, either by aldosterone perfusion or by feeding a salt deficient diet, in Af17 knockout mice completely compensated for Af17 deficiency to restore blood pressure and urinary sodium excretion to those observed in wild type mice ${ }^{44}$.

Potential genetic-epigenetic interplay of Dot1/Af9/Af17 in blood pressure control.

Given the experimental data in model systems, it is reasonable to hypothesize that genetic variation in the components of the Dot1a-mediated epigenetic control of collecting duct $\mathrm{Na}^{+}$ excretion may lead to disorders of renal $\mathrm{Na}+$ excretion, and, thus, potentially hypertensive or hypotensive disorders in humans. Duarte et al. ${ }^{51}$ used a pharmacogenomics approach to test the hypothesis that that genetic variation in the Dot1 epigenetic pathway contributes to the known variability of antihypertensive response to hydrochlorothiazide (HCTZ). These authors investigated associations between genetic variations in DOT1L, AF9, SIRT1, and $S G K 1$ ( $A F^{\prime} 17$ was not studied) and the blood pressure lowering effects of the HCTZ in two large, distinct hypertensive cohorts [Pharmacogenomic Evaluation of Antihypertensive Responses (PEAR); clinicaltrials.gov \#NCT00246519) and the Genetic Epidemiology of Responses to Antihypertensives (GERA); clinicaltrials.gov \#NCT00005520]. The strongest pharmacogenetic association with HCTZ response was observed with the single nucleotide 
polymorphism (SNP) located in intron 7 (rs2269879) in DOT1L, which was only observed in PEAR Caucasians ${ }^{51}$. Because the association did not replicate in GERA, the authors could not exclude the association in PEAR as a chance finding. However, they suggested that one reason for a lack of replication could have been differences in measurement sensitivities between home blood pressure and office blood pressure determinations in the two studies ${ }^{51}$. Nonetheless, the results invite further investigation to replicate the association found in PEAR is warranted.

In an exploratory analysis, Duarte et al. ${ }^{51}$ also evaluated the role of SNPs in the Dot1 pathway on untreated blood pressure in the GERA and PEAR cohorts. They identified rs12350051 in $A F 9$ as being associated with baseline blood pressure in both GERA and PEAR African-Americans. However, in another PEAR and two smaller normotensive cohorts, this association did not replicate. Thus further replication studies are needed to verify potential roles for this polymorphism in human hypertension ${ }^{51}$.

The association of AF17 with human blood pressure has not been analyzed. However, AF17 lies within $290 \mathrm{~kb}$ of a putative blood pressure locus at 17p11-21 around marker D17S250 on human chromosome $17^{5253}$. The syntenic fragments of this region in rat and mice encode an AF17 ortholog and show strong associations with hypertension ${ }^{54,55}$. Given the potent effects of Af7 ablation on blood pressure in mice $^{44}$, detailed genetic association studies humans are warranted.

\section{Conclusion}

The pace of recent advances in the intertwined fields of transcriptional regulatory machinery and epigenetics has been extraordinary, yielding new concepts concerning multiplexed histone modification programs, periodicity of factor recycling and functional events that provide a rich but specific basis for transcriptional regulation of individual genes. Understanding how genes move through distinct repressed, primed, and active chromatin states is a major goal in understanding transcriptional control processes that regulate gene induction. While much emphasis has been placed on the mechanisms of co-activators, much less is known about co-repressors and about removal of co-repressors --- "de-repression" --as transcriptional control mechanisms. As models for transcription have been refined to include sequential steps and periodicity, how co-repressors are released to allow activation of a given gene and then re-engaged to reset the chromatin for subsequent rounds of transcription represents a new frontier. The epigenetic de-repression model for aldosterone activation of $\mathrm{ENaC}$ in the collecting duct thus provides compelling new insights.

The relevance of the Dot1 pathways in collecting duct sodium transport in human health and disease is presently open to conjecture, but the mouse models of CNT/CD-specific Dot1 inactivation of global Af17 ablation suggest potential physiological and clinical relevance in humans. Potentially, dysregulation of the Dot1a system in the collecting duct may lead to disorders of renal $\mathrm{Na}^{+}$excretion, and, thus, abnormal blood pressure phenotypes in humans, much the same as activating or inactivating mutations in $\mathrm{ENaC}$ leads to monogenic forms of $\mathrm{Na}^{+}$retention and hypertension (Liddle's syndrome) or renal $\mathrm{Na}^{+}$wasting and hypotension (pseudohypoaldosteronism type 1$)^{56}$.

Although aldosterone plays a significant role in the development of hypertension and the progression of cardiovascular and renal damage, relatively little is known, compared to other steroid hormones, about how aldosterone signals to chromatin to effect changes in gene transcription. With the increased clinical attention to aldosterone and MR antagonists in the pathogenesis and therapy of chronic cardiovascular and renal diseases, there is a growing gap in understanding the full range of aldosterone effects on the epigenetic and 
transcriptional machinery. With Dot1a now identified as the first chromatin modifier to be regulated by aldosterone, future work will likely identify other aldosterone-sensitive epigenetic readers and effectors of clinical relevance to cardiovascular and renal health and disease. Finally, studies of transcriptional regulation of aENaC over the years have been limited and have raised as many questions as they have answered. MR-dependent and independent control points have been identified but not mechanistically integrated into a comprehensive model, and the role of epigenetic factors in controlling aENaCtranscription is a young science. With the underpinnings in place for such an integrated model, as well as new mouse models to interrogate the system, the field is poised for significant advances.

\section{Acknowledgments}

Financial support for this work: NIH grant R01 DK075065

\section{REFERENCES}

1. Gormley K, Dong Y, Sagnella GA. Regulation of the epithelial sodium channel by accessory proteins. Biochem J. 2003; 371:1-14. [PubMed: 12460120]

2. Schild L. The epithelial sodium channel: from molecule to disease. Rev Physiol Biochem Pharmacol. 2004; 151:93-107. [PubMed: 15146350]

3. Swift PA, MacGregor GA. The epithelial sodium channel in hypertension: genetic heterogeneity and implications for treatment with amiloride. Am J Pharmacogenomics. 2004; 4:161-168. [PubMed: 15174897]

4. Tamura H, Schild L, Enomoto N, Matsui N, Marumo F, Rossier BC. Liddle disease caused by a missense mutation of beta subunit of the epithelial sodium channel gene. J Clin Invest. 1996; 97:1780-1784. [PubMed: 8601645]

5. Bonny O, Knoers N, Monnens L, Rossier BC. A novel mutation of the epithelial Na+ channel causes type 1 pseudohypoaldosteronism. Pediatr Nephrol. 2002; 17:804-808. [PubMed: 12376807]

6. Soundararajan R, Lu M, Pearce D. Organization of the ENaC-regulatory machinery. Crit Rev Biochem Mol Biol. 2012; 47:349-359. [PubMed: 22506713]

7. Debonneville C, Flores SY, Kamynina E, Plant PJ, Tauxe C, Thomas MA, Munster C, Chraibi A, Pratt JH, Horisberger JD, Pearce D, Loffing J, Staub O. Phosphorylation of Nedd4-2 by Sgk1 regulates epithelial $\mathrm{Na}(+)$ channel cell surface expression. Embo J. 2001; 20:7052-7059. [PubMed: 11742982]

8. Mick VE, Itani OA, Loftus RW, Husted RF, Schmidt TJ, Thomas CP. The alpha-subunit of the epithelial sodium channel is an aldosterone-induced transcript in mammalian collecting ducts, and this transcriptional response is mediated via distinct cis-elements in the 5'-flanking region of the gene. Mol Endocrinol. 2001; 15:575-588. [PubMed: 11266509]

9. Stokes JB, Sigmund RD. Regulation of $\mathrm{rENaC}$ mRNA by dietary $\mathrm{NaCl}$ and steroids: organ, tissue, and steroid heterogeneity. Am J Physiol. 1998; 274:C1699-C1707. [PubMed: 9611136]

10. Kohler S, Pradervand S, Verdumo C, Merillat AM, Bens M, Vandewalle A, Beermann F, Hummler E. Analysis of the mouse Scnn1a promoter in cortical collecting duct cells and in transgenic mice. Biochim Biophys Acta. 2001; 1519:106-110. [PubMed: 11406278]

11. Ronzaud C, Loffing J, Bleich M, Gretz N, Grone HJ, Schutz G, Berger S. Impairment of sodium balance in mice deficient in renal principal cell mineralocorticoid receptor. J Am Soc Nephrol. 2007; 18:1679-1687. [PubMed: 17475815]

12. Christensen BM, Perrier R, Wang Q, Zuber AM, Maillard M, Mordasini D, Malsure S, Ronzaud C, Stehle JC, Rossier BC, Hummler E. Sodium and potassium balance depends on alphaENaC expression in connecting tubule. J Am Soc Nephrol. 2010; 21:1942-1951. [PubMed: 20947633]

13. Mellor J. Dynamic nucleosomes and gene transcription. Trends Genet. 2006; 22:320-329. [PubMed: 16631276]

14. Martin C, Zhang Y. The diverse functions of histone lysine methylation. Nat Rev Mol Cell Biol. 2005; 6:838-849. [PubMed: 16261189] 
15. Chen SY, Bhargava A, Mastroberardino L, Meijer OC, Wang J, Buse P, Firestone GL, Verrey F, Pearce D. Epithelial sodium channel regulated by aldosterone-induced protein sgk. Proc Natl Acad Sci U S A. 1999; 96:2514-2519. [PubMed: 10051674]

16. Jenuwein T, Laible G, Dorn R, Reuter G. SET domain proteins modulate chromatin domains in euand heterochromatin. Cell Mol Life Sci. 1998; 54:80-93. [PubMed: 9487389]

17. van Leeuwen F, Gafken PR, Gottschling DE. Dot1p modulates silencing in yeast by methylation of the nucleosome core. Cell. 2002; 109:745-756. [PubMed: 12086673]

18. Ng HH, Feng Q, Wang H, Erdjument-Bromage H, Tempst P, Zhang Y, Struhl K. Lysine methylation within the globular domain of histone $\mathrm{H} 3$ by Dot 1 is important for telomeric silencing and Sir protein association. Genes Dev. 2002; 16:1518-1527. [PubMed: 12080090]

19. Feng Q, Wang H, Ng HH, Erdjument-Bromage H, Tempst P, Struhl K, Zhang Y. Methylation of H3-lysine 79 is mediated by a new family of HMTases without a SET domain. Curr Biol. 2002; 12:1052-1058. [PubMed: 12123582]

20. Min J, Feng Q, Li Z, Zhang Y, Xu RM. Structure of the catalytic domain of human DOT1L, a nonSET domain nucleosomal histone methyltransferase. Cell. 2003; 112:711-723. [PubMed: 12628190]

21. Jones B, Su H, Bhat A, Lei H, Bajko J, Hevi S, Baltus GA, Kadam S, Zhai H, Valdez R, Gonzalo S, Zhang Y, Li E, Chen T. The histone H3K79 methyltransferase Dot1L is essential for mammalian development and heterochromatin structure. PLoS Genet. 2008; 4:e1000190. [PubMed: 18787701]

22. Singer MS, Kahana A, Wolf AJ, Meisinger LL, Peterson SE, Goggin C, Mahowald M, Gottschling DE. Identification of high-copy disruptors of telomeric silencing in Saccharomyces cerevisiae. Genetics. 1998; 150:613-632. [PubMed: 9755194]

23. Nguyen AT, Zhang Y. The diverse functions of Dot1 and H3K79 methylation. Genes Dev. 2011; 25:1345-1358. [PubMed: 21724828]

24. Kim W, Kim R, Park G, Park JW, Kim JE. Deficiency of H3K79 histone methyltransferase Dot1like protein (DOT1L) inhibits cell proliferation. J Biol Chem. 2012; 287:5588-5599. [PubMed: 22190683]

25. Wu H, Chen L, Zhang X, Zhou Q, Li J-M, Berger S, Borok Z, Zhou B, Xiao Z, Yin H, Liu M, Wang Y, Jin J, Blackburn MR, Xia Y, Zhang W. Aqp5 Is a new transcriptional target of Dot1a and a regulator of Aqp2. PLoS One. 2013; 8:e53342. [PubMed: 23326416]

26. Zhou Q, Liu K, Wu H, Chen L, Pouranan V, Yuan M, Xiao Z, Peng W, Xiang A, Tang R, Zhang W. Spironolactone rescues Dot1a-Af9-mediated repression of endothelin-1 and improves kidney injury in streptozotocin-induced diabetic rats. PLoS One. 2012; 7:e47360. [PubMed: 23077601]

27. Yu Z, Kong Q, Kone BC. CREB trans-activation of disruptor of telomeric silencing-1 mediates forskolin inhibition of CTGF transcription in mesangial cells. Am J Physiol Renal Physiol. 2010; 298:F617-F624. [PubMed: 20053791]

28. Zhang W, Hayashizaki Y, Kone BC. Structure and regulation of the mDot1 gene, a mouse histone H3 methyltransferase. Biochem J. 2004; 377:641-651. [PubMed: 14572310]

29. Zhang W, Xia X, Jalal DI, Kuncewicz T, Xu W, Lesage GD, Kone BC. Aldosterone-sensitive repression of ENaCalpha transcription by a histone $\mathrm{H} 3$ lysine-79 methyltransferase. Am J Physiol Cell Physiol. 2006; 290:C936-C946. [PubMed: 16236820]

30. Reisenauer MR, Anderson M, Huang L, Zhang Z, Zhou Q, Kone BC, Morris AP, Lesage GD, Dryer SE, Zhang W. AF17 competes with AF9 for binding to Dot1a to up-regulate transcription of epithelial Na+ channel alpha. J Biol Chem. 2009; 284:35659-35669. [PubMed: 19864429]

31. Wu H, Chen L, Zhou Q, Zhang W. AF17 facilitates Dotla nuclear export and upregulates ENaCmediated $\mathrm{Na}+$ transport in renal collecting duct cells. PLoS One. 2011; 6:e27429. [PubMed: 22087315]

32. Zhang W, Yu Z, Wu H, Chen L, Kong Q, Kone BC. An Af9 cis-element directly targets Dot1 to mediate transcriptional repression of the alphaENaC gene. Am J Physiol Renal Physiol. 2012

33. Zhang W, Xia X, Reisenauer MR, Hemenway CS, Kone BC. Dot1a-AF9 complex mediates histone H3 Lys-79 hypermethylation and repression of ENaCalpha in an aldosterone-sensitive manner. J Biol Chem. 2006; 281:18059-18068. [PubMed: 16636056] 
34. Zhang W, Xia X, Reisenauer MR, Hemenway CS, Kone BC. Dot1a-AF9 Complex Mediates Histone H3 Lys-79 Hypermethylation and Repression of ENaC $\{$ alpha $\}$ in an Aldosteronesensitive Manner. J Biol Chem. 2006; 281:18059-18068. [PubMed: 16636056]

35. Reisenauer MR, Wang SW, Xia Y, Zhang W. Dot1a contains three nuclear localization signals and regulates the epithelial $\mathrm{Na}+$ channel $(\mathrm{ENaC})$ at multiple levels. Am J Physiol Renal Physiol. 2010; 299:F63-F76. [PubMed: 20427473]

36. Iida S, Seto M, Yamamoto K, Komatsu H, Tojo A, Asano S, Kamada N, Ariyoshi Y, Takahashi T, Ueda R. MLLT3 gene on 9p22 involved in $\mathrm{t}(9 ; 11)$ leukemia encodes a serine/proline rich protein homologous to MLLT1 on 19p13. Oncogene. 1993; 8:3085-3092. [PubMed: 8414510]

37. Collins EC, Appert A, Ariza-McNaughton L, Pannell R, Yamada Y, Rabbitts TH. Mouse Af9 is a controller of embryo patterning, like Mll, whose human homologue fuses with Af9 after chromosomal translocation in leukemia. Mol Cell Biol. 2002; 22:7313-7324. [PubMed: 12242306]

38. Pramparo T, Grosso S, Messa J, Zatterale A, Bonaglia MC, Chessa L, Balestri P, Rocchi M, Zuffardi O, Giorda R. Loss-of-function mutation of the AF9/MLLT3 gene in a girl with neuromotor development delay, cerebellar ataxia, and epilepsy. Hum Genet. 2005; 118:76-81. [PubMed: 16001262]

39. Haribaskar R, Putz M, Schupp B, Skouloudaki K, Bietenbeck A, Walz G, Schafer T. The planar cell polarity (PCP) protein Diversin translocates to the nucleus to interact with the transcription factor AF9. Biochem Biophys Res Commun. 2009; 387:212-217. [PubMed: 19591803]

40. Zhang W, Xia X, Reisenauer MR, Rieg T, Lang F, Kuhl D, Vallon V, Kone BC. Aldosteroneinduced Sgk1 relieves Dot1a-Af9-mediated transcriptional repression of epithelial $\mathrm{Na}$ channel alpha. J Clin Invest. 2007; 117:773-783. [PubMed: 17332896]

41. Lin JJ, Hemenway CS. Hsp90 directly modulates the spatial distribution of AF9/MLLT3 and affects target gene expression. J Biol Chem. 2010; 285:11966-11973. [PubMed: 20159978]

42. Zhang Z, Huang L, Reisenauer MR, Wu H, Chen L, Zhang Y, Xia Y, Zhang W. Widely expressed Af17 is likely not required for embryogenesis, hematopoiesis, and animal survival. Genesis. 2010; 48:693-706. [PubMed: 21170927]

43. Lin YM, Ono K, Satoh S, Ishiguro H, Fujita M, Miwa N, Tanaka T, Tsunoda T, Yang KC, Nakamura Y, Furukawa Y. Identification of AF17 as a downstream gene of the beta-catenin/T-cell factor pathway and its involvement in colorectal carcinogenesis. Cancer Res. 2001; 61:6345-6349. [PubMed: 11522623]

44. Chen L, Wu H, Pochynyuk OM, Reisenauer MR, Zhang Z, Huang L, Zaika OL, Mamenko M, Zhang W, Zhou Q, Liu M, Xia Y, Zhang W. Af17 deficiency increases sodium excretion and decreases blood pressure. J Am Soc Nephrol. 2011; 22:1076-1086. [PubMed: 21546577]

45. Rahman S, Islam R. Mammalian Sirt1: insights on its biological functions. Cell Commun Signal. 2011; 9:11. [PubMed: 21549004]

46. Knight JR, Milner J. SIRT1, metabolism and cancer. Curr Opin Oncol. 2012; 24:68-75. [PubMed: 22080944]

47. Zhang D, Li S, Cruz P, Kone BC. Sirtuin 1 functionally and physically interacts with disruptor of telomeric silencing-1 to regulate alpha-ENaC transcription in collecting. duct J Biol Chem. 2009; 284:20917-20926.

48. Stockand JD. New ideas about aldosterone signaling in epithelia. Am J Physiol Renal Physiol. 2002; 282:F559-F576. [PubMed: 11880316]

49. Zhou ZH, Bubien JK. Nongenomic regulation of ENaC by aldosterone. Am J Physiol Cell Physiol. 2001; 281:C1118-C1130. [PubMed: 11546647]

50. Pearce D, Kleyman TR. Salt, sodium channels, and SGK1. J Clin Invest. 2007; 117:592-595. [PubMed: 17332888]

51. Duarte JD, Zineh I, Burkley B, Gong Y, Langaee TY, Turner ST, Chapman AB, Boerwinkle E, Gums JG, Cooper-Dehoff RM, Beitelshees AL, Bailey KR, Fillingim RB, Kone BC, Johnson JA. Effects of genetic variation in H3K79 methylation regulatory genes on clinical blood pressure and blood pressure response to hydrochlorothiazide. J Transl Med. 2012; 10:56. [PubMed: 22440088]

52. Mansfield TA, Simon DB, Farfel Z, Bia M, Tucci JR, Lebel M, Gutkin M, Vialettes B, Christofilis MA, Kauppinen-Makelin R, Mayan H, Risch N, Lifton RP. Multilocus linkage of familial 
hyperkalaemia and hypertension, pseudohypoaldosteronism type II, to chromosomes 1q31-42 and 17p11-q21. Nat Genet. 1997; 16:202-205. [PubMed: 9171836]

53. Levy D, DeStefano AL, Larson MG, O’Donnell CJ, Lifton RP, Gavras H, Cupples LA, Myers RH. Evidence for a gene influencing blood pressure on chromosome 17. Genome scan linkage results for longitudinal blood pressure phenotypes in subjects from the framingham heart study.

Hypertension. 2000; 36:477-483. [PubMed: 11040222]

54. Dukhanina OI, Dene H, Deng AY, Choi CR, Hoebee B, Rapp JP. Linkage map and congenic strains to localize blood pressure QTL on rat chromosome 10. Mamm Genome. 1997; 8:229-235. [PubMed: 9096100]

55. Salzler HR, Griffiths R, Ruiz P, Chi L, Frey C, Marchuk DA, Rockman HA, Le TH. Hypertension and albuminuria in chronic kidney disease mapped to a mouse chromosome 11 locus. Kidney Int. 2007; 72:1226-1232. [PubMed: 17851470]

56. Hummler E. Epithelial sodium channel, salt intake, and hypertension. Curr Hypertens Rep. 2003; 5:11-18. [PubMed: 12530930] 


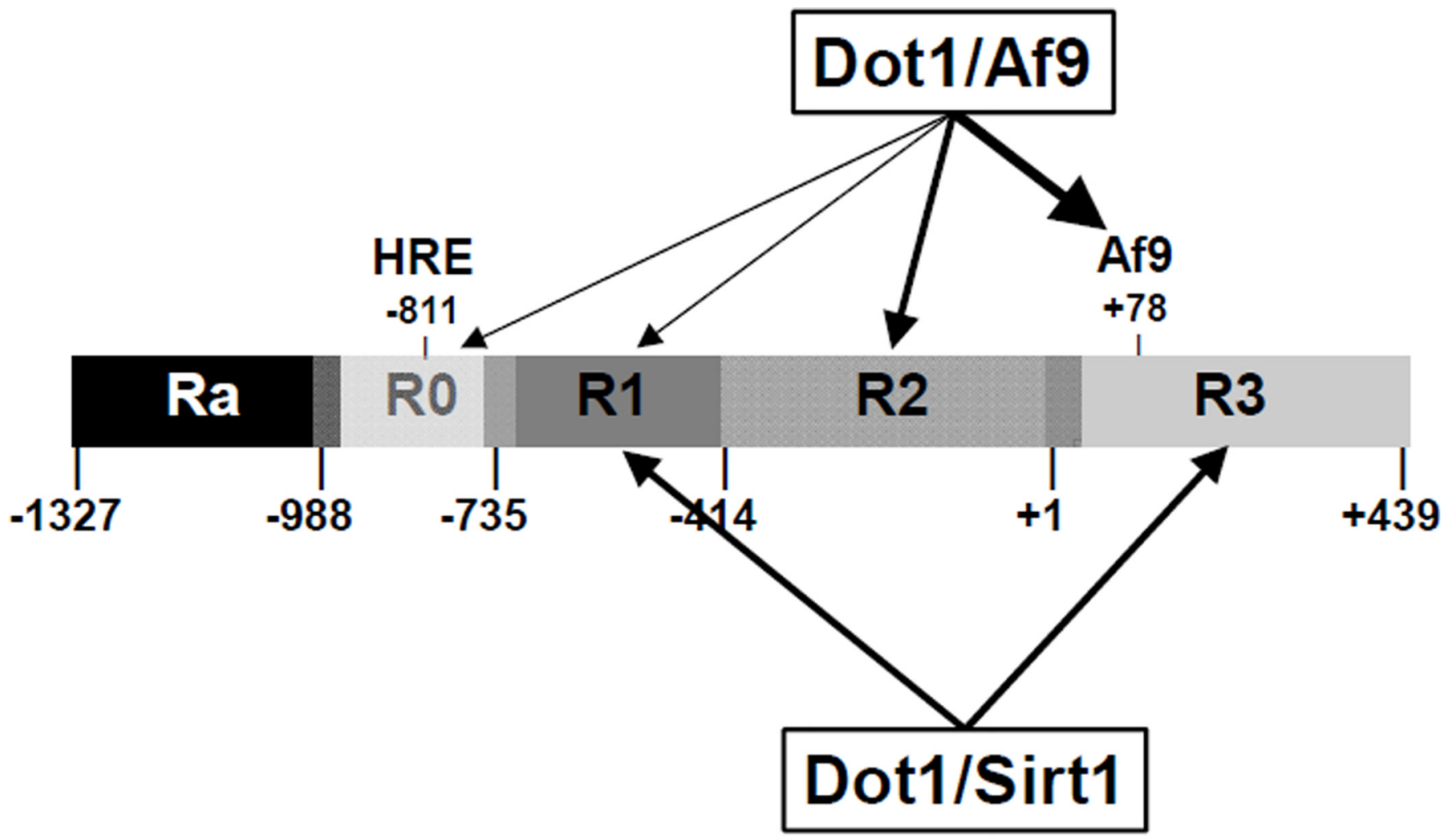

\section{$\langle$ ENaC promoter subregions basal conditions}

Figure 1.

Schematic model of key regulatory control points of the aENaC promoter. Five subregions of the $\mathrm{a} E N a C$ promoter (Ra-R3) used in experimental studies are indicated by shading and nucleotide positions. The hormone response element (HRE) beginning at -811 to which the liganded mineralocorticoid receptor binds, and the Af9 element beginning at +78 , to which Af9 and its protein partner Dot1 bind, are indicated. The occupancy of Dot1a/Af9 and Dot1a/Sirt 1 complexes along the $\mathrm{a} E N a C$ promoter, as discerned by sequential chromatin immunoprecipitation studies, is depicted with arrows. The weight of the arrows indicates the relative abundance of the complex at each subregion under basal conditions. 


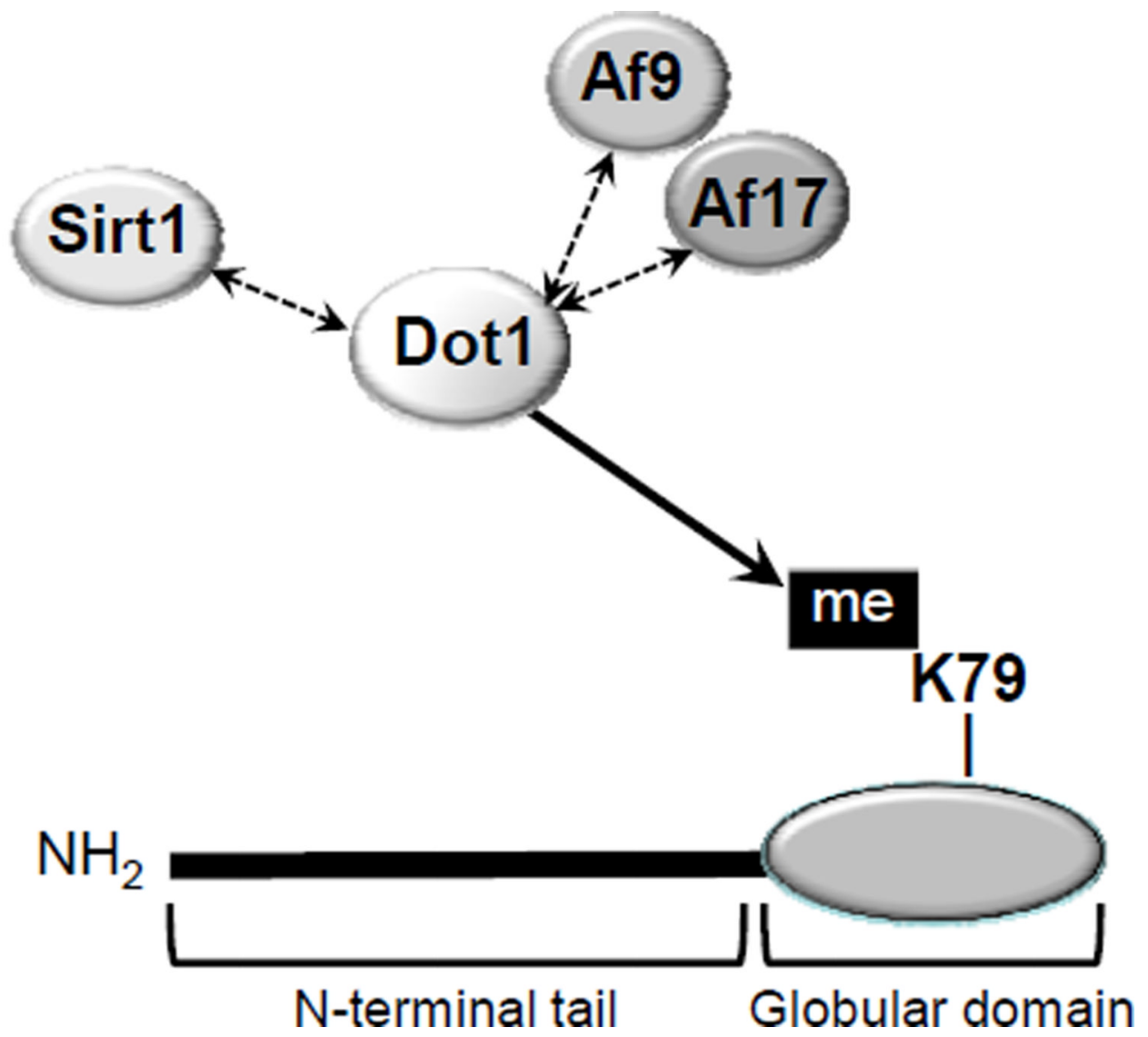

Figure 2.

Dot1, its protein partners, and histone H3. Dot1a specifically methylates ("me") K79 in the globular domain of histone H3. Dot1a can reversibly bind Sirt1 or separately, through competition for a common binding site on Dot1a, Af9 or Af17. The interaction with Sirt1 appears to enhance the distributive activity of Dot1a to methylate H3 K79, whereas the interactions with Af9 and Dot1 appear to regulate the nuclear-cytoplasmic distribution of Dot1a and its targeting to the Af9 cis-element of the aENaC promoter (see text for details). 
basal

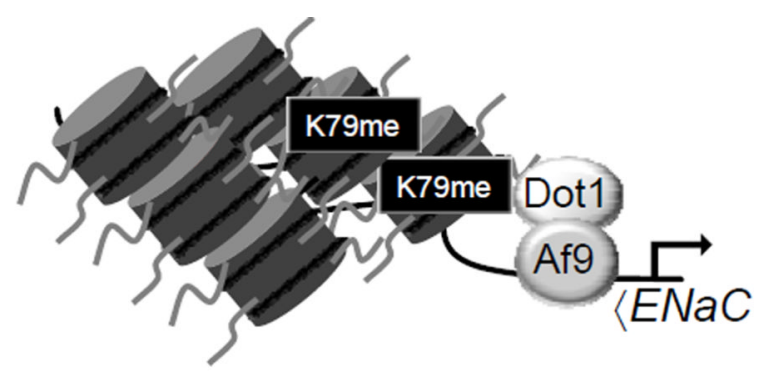

Closed chromatin transcriptional repression

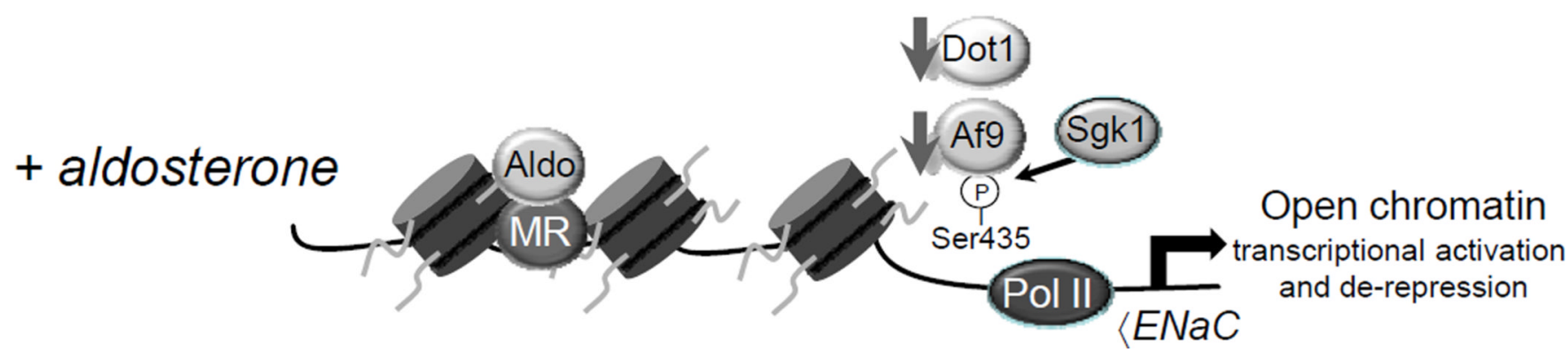

Figure 3.

Model of basal Dot1a/Af9-mediated transcriptional repression and aldosterone- and Sgk1induced transcriptional de-repression and activation of the $\alpha E N a C$ gene in the collecting duct. Transcriptionally silent or less active genes commonly have a closed chromatin structure, marked by histone modifications such as methylation of histone H3 K79 (K79me). Transcriptionally active genes have an open chromatin structure that allows access of the DNA to the general transcription factors and various co-activator proteins. Under basal conditions, the histone $\mathrm{H} 3$ methyltransferase Dot 1 a is complexed with the DNA-binding protein Af9, which targets the complex to an Af9 binding element in aENaCgene, and thereby facilitates the ability of Dot1a to hypermethylate K79 of histone $\mathrm{H} 3$ associated with the $\mathrm{a} E N \mathrm{NC}$ promoter. This action establishes a closed chromatin configuration that constrains aENaC transcription. Aldosterone (aldo) downregulates the expression of both Dot1a and Af9 leading to decreased abundance of the repressor complex. Aldosterone also induces Sgk1, which phosphorylates Ser435 of Af9, causing disruption of the proteinprotein interactions of Dot1a and Af9, and dispersal of Dot1a from the aENaCpromoter. Hypomethylation of histone $\mathrm{H} 3 \mathrm{~K} 79$ results, and transcriptional constraints on $\mathrm{a} E \mathrm{NaC}$ are released. At a separate region of the $\mathrm{a} E N \mathrm{NaC}$ promoter, the aldosterone-liganded mineralocorticoid receptor (MR) binds to its cognate hormone response element to transactivate the aENaC gene. 

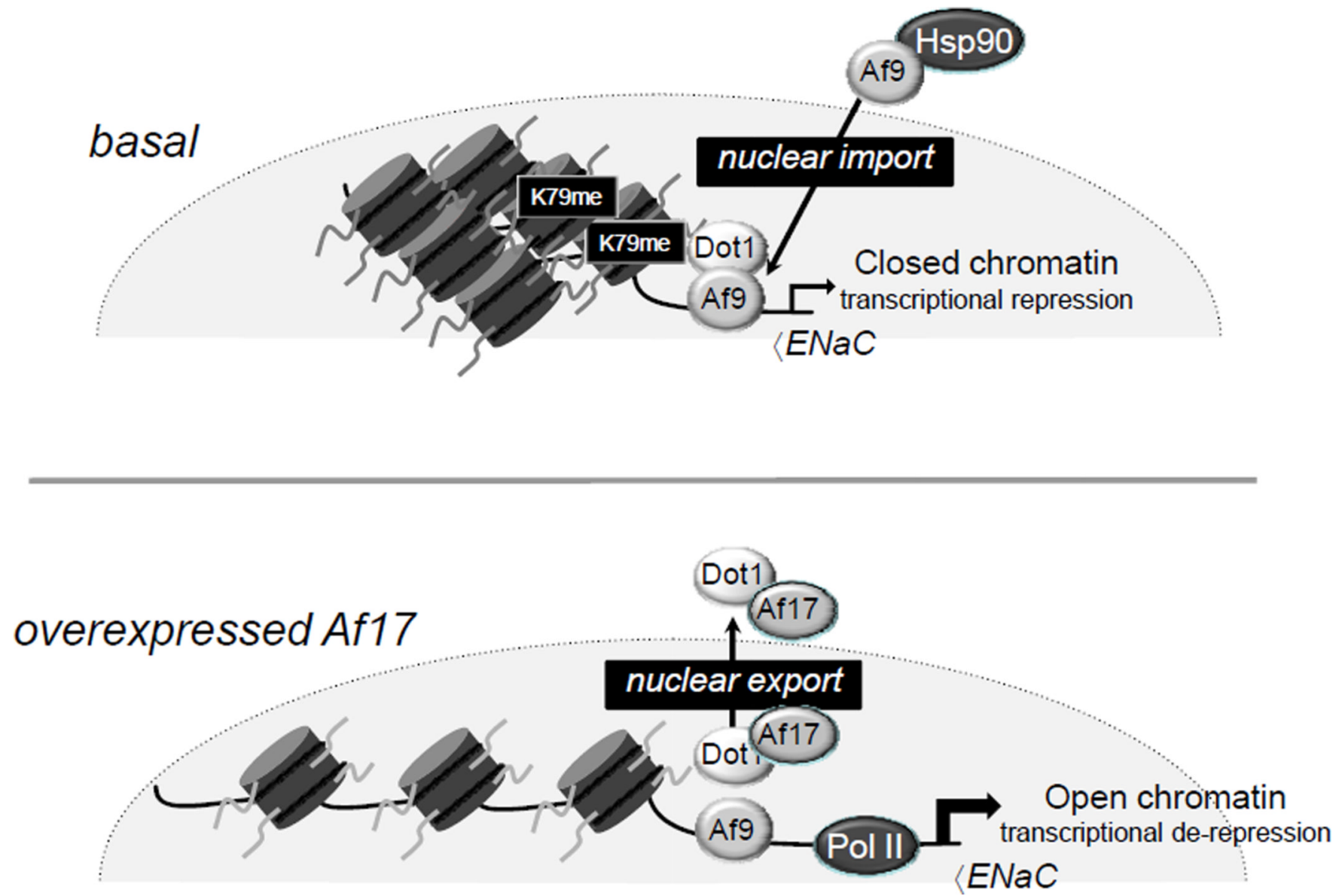

Figure 4.

Role of nuclear-cytoplasmic trafficking in control of Dot1 epigenetic control of $a E N a C$ transcription. Under basal conditions, the targeting of the Dot1a/Af9 complex to the $a E N a C$ promoter and its suppression of a $E N a C$ transcription is controlled, at least in part, by Hsp90 chaperoning of Af9 to the nucleus, and Af9 binding (at the expense of Af17) to Dot1a. Experimental manipulation of Af17 expression suggests that Af17 displacement of Af9 from Dot1a results in the latter's export from the nucleus via a leptomycin-sensitive mechanism. As a result, Dot1a-mediated hypermethylation of histone H3 K79 (K79me) is diminished, and repression of aENaC transcription is lifted. 